\title{
Mig-RL: a Natural Preventive Treatment against Migraine. Outcomes of a Randomized, Double-Blind Clinical Trial
}

\author{
Rémi Shrivastava $^{1,2 *}$, Gisela DA Silva Borges ${ }^{1}$, François Gabrielli' ${ }^{1}$, Amélie Descheemaeker ${ }^{1}$, Ravi Shrivastava ${ }^{2}$ \\ ${ }^{1}$ Clermont Université, université d'Auvergne, Neuro-Dol, BP 10448, F-63000, Clermont-Ferrand \& Inserm U1107, F-63001 Clermont-Ferrand, \\ France
}

${ }^{2}$ VITROBIO SAS \& NATURVEDA SAS, laboratoire pharmaceutique

*Corresponding Author: Rémi Shrivastava, Clermont Université, université d'Auvergne, Neuro-Dol, BP 10448, F-63000, Clermont-Ferrand \& Inserm U1107, F-63001 Clermont-Ferrand, France

Received date: June 15, 2021; Accepted date: June 29, 2021; Published date: July 05, 2021

Citation: Rémi Shrivastava., Gisela DA Silva Borges., François Gabrielli., Amélie Descheemaeker., Ravi Shrivastava. (2021) Mig-RL: a Natural Preventive Treatment against Migraine. Outcomes of a Randomized, Double-Blind Clinical Trial. J. Neuroscience and Neurological Surgery. 8(7); DOI:10.31579/2578-8868/192

Copyrighst: (c) 2021 Rémi Shrivastava, This is an open-access article distributed under the terms of The Creative Commons Attribution License, which permits unrestricted use, distribution, and reproduction in any medium, provided the original author and source are credited

\begin{abstract}
Background: The efficacy and safety of Mig-RL (Commercial name HERBA MIG), a synergistic association of two plant extracts, Tanacetum parthenium (150mg, 0.5\% parthenolide) and Salix Alba (150mg, 25\% salicin) were investigated as a preventive treatment for episodic migraine. Mig-RL was compared with a placebo in a randomized, double-blind clinical trial.

Materials and Methods: This trial included 129 patients having episodic migraine and diagnosed with migraines without aura for over a year and meeting the migraine criteria, as defined in the International Classification of Headache Disorder (ICHD-3 :1.1). P). Baseline data was collected for 28 days before the start of the three-months treatment period. Patients were randomized in a 1:1 ratio to receive either Mig-RL (a single dose of 300mg per day, per os) or placebo (identical capsules containing $300 \mathrm{mg}$ starch, 1 per day, per os) for a period of 12 consecutive weeks. The primary endpoint was the mean change in the average number of migraine days per month, comparing the baseline 28-days pre-intervention period with the weeks 9 to 12 after the first dose of the trial regimen. Secondary endpoints were the percentage of patients with a reduction of at least $30 \%$ in the average number of migraine days per month and days of use of any acute headache medication per month. The HIT-6 and MIDAS scores were also evaluated vs. baseline between the two groups.
\end{abstract}

Results: Out of 172 patients enrolled, 129 were randomly assigned to the Mig-RL arm ( $\mathrm{n}=65$ ) or the placebo arm ( $\mathrm{n}=64)$. The mean $( \pm \mathrm{SD})$ number of baseline migraine days per month was $8.4 \pm 1.9$ and $8.7 \pm 1.9$, respectively. The mean reduction in the average number of migraine days per month was $2.5 \pm 0.4(p<0.001)$ with Mig-RL and $1.9 \pm 0.4(p<0.001)$ with placebo. A difference of $0.6 \pm 0.4(p=0.01)$ between the two groups.

The percentage of patients with at least 30\% reduction in average number of migraine days per month was $49 \%$ in Mig-RL and $32 \%$ in placebo ( $p<0.05$ vs. placebo). Only a few isolated and minor side effects were reported and overall Mig-RL was well tolerated by patients.

Conclusions: Mig-RL, a synergistic combination of two plant extracts, seems to have a moderate effect in the prevention of migraines. Without side effects, the combination of Tanacetum parthenium and Salix alba could be an additional help for some patients. However, further investigations and an improvement in the quality of the plants are still necessary.

Key-words: migraine; tanacetum parthenium; salix alba

\section{Introduction}

Migraine is a chronic neurological disease characterized by the presence of several incapacitating neurological symptoms, such as headaches, sensitivity to light and sound, and nausea. Migraine can be episodic or chronic, based on the number of headaches days per month [1]. Episodic migraine is described as migraine with or without aura occurring in a headache pattern of <14 days per month [2]. Migraine without aura manifests in attacks lasting 4 to 72 hours; the headaches are characterized by their unilateral location, pulsating quality, moderate or severe intensity, aggravated by routine physical activity and associated with nausea and/or photophobia and phonophobia [1]. Migraine with aura is primarily characterized by the focal neurological symptoms that usually precede the headache. Chronic migraine, on the other hand, refers to patients manifesting long-duration headaches ( $\geq 15$ days / month) [3].

Migraine affects over 1 billion individuals worldwide, making migraine the third leading cause of disability worldwide [4] Migraine symptoms interfere with normal day-to-day life, including family, education, work, 
and contributing to the development of comorbidities such as cardiovascular disease, depression and anxiety [5]

Migraine management is based either on non-pharmacological approaches, such as suppressing triggering factors (if they are known), or pharmacological approaches, such as abortive (acute) treatment and/or preventive (prophylactic) treatment [6]. The recommendations for using pharmacological migraine prevention vary greatly between published treatments guidelines. As a general rule, migraine prevention should be considered when attacks affect quality of life and is indicated in roughly one third of migraine patients [6], [7]. The comorbidities should be taken into account when considering and selecting an approach to prevent migraine attacks.

The following classes of medications are used for migraine prevention: antiepileptic drugs, antidepressants, beta blockers, injectable calcitonin gene-related peptide (CGRP) monoclonal antibodies, calcium channel antagonists, serotonin antagonists, botulinum neurotoxins, NSAIDs, and others including cyanocobalamin, riboflavin, tryptophan and magnesium [6]. A drug is chosen based on its efficacy, its safety profile, the patient's preference, and the presence of any coexistent or comorbid conditions. All these therapies are symptomatic, often have insufficient efficacy, and may have variable side effects [8]. Part of current research is now targeting herbal products to treat migraine patients (adults and children) [9]. Because herbs, spices, and their active constituents can influence many processes associated with migraine pathophysiology, they can be used as an alternative or adjunct intervention to enhance current treatment outcomes [10]. Moreover, their natural aspects make them more accepted by the patients, as they usually induce fewer side effects compared to traditional chemical drugs.

Tanacetum parthenium L. belongs to the Asteraceae family and is a perennial herb commonly known as Feverfew. Tanacetum parthenium contains many sesquiterpene lactones, where the most abundant is the parthenolide (up to $85 \%$ of the total sesquiterpene content), in the leaves and the flower heads [11]. However, flower heads and leaves differ in quali-quantitative component profile (e.g. parthenolide is higher in the flower heads than in the leaves) [12]. The plant also contains a high percentage of sterols and triterpenes in the roots [13]. Its medical properties against inflammation and vertigo have been known for a long time and Tanacetum parthenium is traditionally used as an antipyretic and for the treatment of arthritis, headaches, insect bites, spasms, and menstrual disorders [13]. More recently, Tanacetum parthenium has been extensively studied for migraine treatment and prophylaxis. The results obtained so far corroborate its efficacy [14]. Antinociceptive, antiinflammatory and anti-serotonin features were preclinically described [15]-[17]. As mentioned above, therapeutic effects are generally attributed to leaves and/or flowers. This is mainly due to the presence of sesquiterpene lactones and flavonoids, which are also found in abundance in the plant[13]. The lipophilic character of parthenolide seems to enhance particularly well the passage through the blood brain barrier [18]. Tanacetum parthenium also shows a good safety profile) [19].

In the past, Shrivastava et al. have shown, in a prospective pilot openlabel study, the prophylactic efficacy of a combination of Tanacetum parthenium (150mg) and Salix alba $(150 \mathrm{mg}$ ) when administered twice a day (Mig-RL@) [20] The latter is traditionally recognized for its analgaesic effects, mainly due to the presence of salicin, precursor of salicylic acid (aspirin). To confirm these results with a more robust methodology and to study the efficacy of a slightly optimized formula of Mig-RL $®$, we conducted a 16-weeks, double-blind, randomized, placebocontrolled clinical study to evaluate the efficacy, safety and side effects of the association of Tanacetum parthenium $(150 \mathrm{mg}$ with at least $0.5 \%$ of parthenolide) and Salix alba (150 mg with at least $25 \%$ of salicin) plant extracts, administered once a day (Mig-RL®, VITROBIONATURVEDA, France) [20].

\section{Materials and Methods:}

\section{Trial oversight}

The protocol was approved by relevant ethics committees (Ashirwad Ethics Committee) and institutional review boards. While conducting the study, the authors vouch for the adherence to the protocol, the accuracy and completeness of data and analyses, and the report of adverse events. The trial complied with the International Conference on Harmonisation Guidelines for Good Clinical Practice, the principles of the Declaration of Helsinki, relevant national and local regulations. At the time of screening, participants signed consent forms. The trial sponsor, VITROBIO NATURVEDA SAS, provided the trial medication and performed the data analysis. An independent organization Pharmazone (CIN: U51397AS2014PTC011790) was commissioned to audit and validate the proper respect of the processes established by the protocol throughout the duration of the study. After validation, Pharmazone was responsible for forwarding the anonymised results to the sponsor.

\section{Trial Participants}

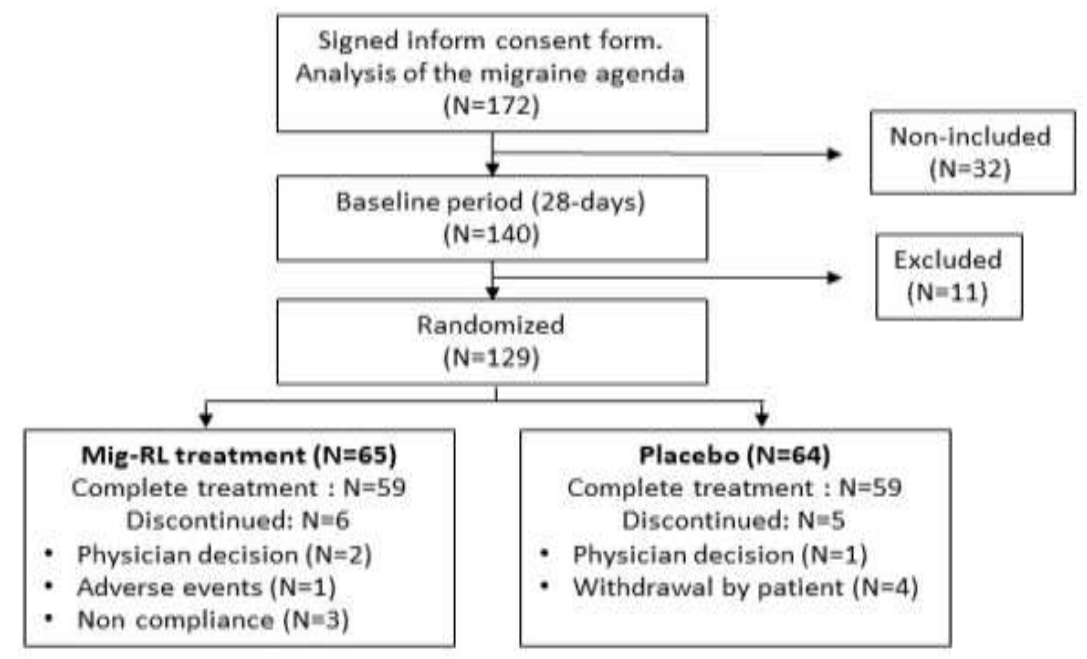

Figure 1: Study Flow of Mig-RL study 
This double-blind, randomized, placebo-controlled study was performed at the Mudra ClinCare Hospital in Mumbai, India. The first patient was recruited in June 2017 and the last patient monitored was in May 2019. The patients eligible to enter this study were aged between 18 and 55 years old, males or females [21]. All patients had been diagnosed for over a year with migraines without aura and met the definition of the International Classification of Headache Disorder version 3 [2]. 28 days of observation before the start of the three-months treatment period was used to establish the baseline. To maintain group homogeneity, patients with less than five days of migraine a month or migraine attacks lasting less than four hours were not included in the study. Participants were asked to keep a migraine diary for more than six months to evaluate the frequency of migraines and medication use. Patients that had been using a new treatment for their attacks less than six months prior to the study were not retained, nor were patients who were diagnosed headaches induced by medication overuse. Crisis treatments were authorized to the patients during the study period only if they had been using it for more than six months. All treatments with aspirin, salicylates or herbal extracts were prohibited during the study.

The main non-inclusion criteria were allergies to salicylates and camphor or hypersensitivity to study medication, drug abuse or dependency, chronic psychiatric or systemic diseases, pregnant women, breastfeeding women. Subjects taking antidepressants, neuroleptics, anxiolytics or prophylactic treatment for migraine within three months before the start of the study, were also excluded. The exclusion criteria were chosen for a safety matter and to avoid bias of the final results.

\section{Trial End Points}

The primary end point was the mean change in the average number of migraine days per month, comparing the baseline 28 -day pre-intervention period with the weeks 9-12 after the first dose of the trial regimen [22].

Secondary end points were to compare the average number of migraine days per month, in between the baseline 28-day pre-intervention period and the weeks $1-4$ and 5-8 after the first dose of the trial regimen.

Secondary end point were also to determine the percentage of patients experiencing a reduction from at least $30 \%$ to $50 \%$ on their average migraine days per month, and the mean change in between the baseline on the average number of days per month in which acute migraine medication was used and the weeks 9 to 12 .

A migraine day was defined as any day on which the patient had a migraine or probable migraine. Defined in a calendar day, in which headache pain lasted, at least, 4 consecutive hours and met criteria for migraine or probable migraine (subtype in which only one migraine criterion is absent), or a calendar day in which acute migraine-specific medication was used to treat a headache of any duration [22].

Other secondary end points included the mean change in the score on the six-item Headache Impact Test (HIT-6) and the Migraine Disability Assessment (MIDAS).

HIT-6 and MIDAS tests were designed to provide a global measure of adverse headache impact. HIT-6: scores range from 36 to 78 , with higher scores indicating a greater degree of headache-related disability. MIDAS scores are interpreted as grade $\mathrm{I}=0-5$ (minimal or infrequent disability), grade II $=6-10$ (mild or infrequent disability), grade III $=11-20$ (moderate disability), grade IVa $=21-40$ and higher (severe disability), grade $\mathrm{IVb}=41$ and higher (very severe disability) with higher scores indicating greater disability and decreased scores consistent with improvement [23], [24].

Safety and side-effect profiles were evaluated according to reported adverse events, vital signs (systolic and diastolic blood pressure, pulse, body temperature, and respiratory rate), physical examination, cardiovascular, respiratory system, gastrointestinal system, musculoskeletal and nervous system examination.

\section{Study design}

This randomized, double-blind, placebo-controlled trial consisted of a screening visit, a 28-day pre-intervention period, and a 12-week intervention period, with a final evaluation in week 12 [22]. Based on the screening visit and information collected in a daily diary during the preintervention period, patients were enrolled or excluded from the trial.

Patients satisfying all the ICHD-3 inclusion criterias and none of the exclusion criterias were enrolled and randomly allocated to a 1:1 ratio as per randomization schedule to receive Mig-RL or placebo. Randomization was performed by using SAS Version 9.1.3. The randomization schedule was generated with block randomization methodology.

Patients were seen at five scheduled visits for protocol-specified evaluations: at screening, baseline, week 4 , week 8 , and week 12 , or at the time of early withdrawal from the trial. Patients who withdrew prematurely had final protocol-specified evaluations performed as soon as possible after withdrawal. Headache data (e.g., occurrence, duration, and pain severity; occurrence of photophobia, phonophobia, nausea, or vomiting; and any use of migraine medication) were captured daily through an individual headache diary.

\section{Treatment}

As per the randomization, patients received either Mig-RL or the placebo. Mig-RL treatment group received capsules containing standardized powdered extract of Tanacetum parthenium $150 \mathrm{mg}$ and Salix alba $150 \mathrm{mg}$, characterized by chromatography according to the European Pharmacopoeia, with clear instructions of use (one capsule / day; per os). The extracts contained in the capsules were supplied by the laboratory VITROBIO-NATURVEDA according to their extraction process: water/ethanol (70/30) for a ratio plant extract 3-4/1. The parthenolide content of Tanacetum parthenium was at least $0.5 \%$ and the salicin content in Salix alba was at least $25 \%$. Both plant extracts are listed in the European Pharmacopoeia and are authorized for oral administration to human beings by the European Union Regulatory Authorities. Placebo patients received starch containing capsules $(300 \mathrm{mg})$, with the same instruction as the treatment group (one capsule / day: per os).

\section{Statistical analysis}

Estimations based on the observational trial of Mig-RL in episodic migraine [20] and the Cochrane systemic review to evaluate the efficacy of Tanacetum parthenium as a preventive treatment for migraine [14] predicted that a sample of 80 patients who had completed the trial and could be evaluated, would provide $90 \%$ power to detect a mean $( \pm \mathrm{SD})$ difference of $1.4 \pm 7.2$ in the average number of migraine days per month. With an anticipated rate of 50\%, 130 participants were planned for randomization in this trial. Analyses were conducted in the modified intention- to-treat population, which included all randomly assigned patients who received at least one dose of a trial regimen and had at least 10 days of postbaseline efficacy assessments regarding the primary end point. Safety analyses included all randomly assigned patients who received at least one dose of a trial regimen. Demographic and baseline characteristics were summarized descriptively with a Student's test for comparison between the two groups and Fisher's exact test for analysis of contingencies. The primary efficacy outcome was analysed with two-way repeated measure ANOVA followed by the post hoc Bonferroni's test. The mean change from the baseline with standard errors $( \pm \mathrm{SD})$ is presented for each treatment group, and the difference versus placebo with $95 \%$ confidence interval (CI). For management of missing data in the primary analysis, the average number of headache days per month 
during the 12-week period was prorated to a 28 -day equivalent with the use of all postbaseline observations. The same analyses were used for relevant secondary end points. For the percentage of patients with a reduction of at least 30 to $50 \%$ in the average number of headache days per month, the Cochran-Mantel-Haenszel test was used. $P<0.05$ was considered statistically significant. Adverse events data are collected during the double-blind, placebo-controlled intervention period. The safety population included all the patients who underwent randomization performed by Chi-square test for comparison of adverse events between the two groups.

The analyses were carried out with the software GraphPad Prism version 8.4.2, (La Jolla, USA).

\section{Results:}

\section{Primary end points}

After the analysis of their migraine diary, 140 patients were included, for the 28-day observation period. 11 patients were excluded following doctors' decisions, due to a discrepancy between the results presented in and received at least one dose of a trial regimen. Statistical analysis was

the migraine diary and the data collected after the 28-day observation phase.

A total of 129 patients were randomly assigned to either the Mig-RL group $(n=65)$ or the placebo group $(n=64) .118$ patients received treatments and were included in the study population (Figure 1). A total of 11 patients $(9 \%)$ across the full duration of the study (Mig-RL, $n=6$; placebo, $n=5$ ) discontinued treatment early. The most frequently reported reasons for early discontinuation were withdrawal by patients $(n=4)$, noncompliance to the protocol $(n=3)$, physician's decision for suspected pregnancy $(n=1)$, overuse of other medications $(n=1)$, and adverse events $(n=1)$. Overall, 118 of 129 patients $(91 \%)$ remained in the study until week 12. The demographic data in Table 1 indicated no significant difference between groups.

Mig-RL demonstrated statistically significant reduction from the baseline in the frequency of migraine days during weeks 9-12 compared to placebo $(-0.6 \pm 0.4$ [95\% confidence interval (CI) -1.26 to -0.09 ], $p=0.01$ vs placebo) (Table 2 and Figure 2). Mean of migraine days at baseline (during the 28-day screening period) was $8.4 \pm 1.9$ in Mig-RL group and $8.7 \pm 1.9$ in the placebo group. During weeks $9-12$, mean of migraine days was $5.7 \pm 2.5$ and $6.7 \pm 2.0$ respectively.

\begin{tabular}{|c|c|c|c|}
\hline Characteristic & $\operatorname{Mig}-R L(n=65)$ & Placebo $(n=64)$ & $p$-value \\
\hline Male, n (\%) & $30(23.3)$ & $32(24.8)$ & 0.86 \\
\hline Female, $\mathrm{n}(\%)$ & $35(27.1)$ & $33(25.6)$ & 0.86 \\
\hline Mean age, years & $31.6 \pm 7.7$ & $32.8 \pm 9.4$ & 0.42 \\
\hline Mean weight, kg & $65.8 \pm 7.8$ & $66.2 \pm 6.3$ & 0.74 \\
\hline Mean height, $\mathrm{cm}$ & $163.7 \pm 7.6$ & $164 \pm 7.3$ & 0.59 \\
\hline Mean no. of migraine days at baseline & $8.4 \pm 1.9$ & $8.7 \pm 1.9$ & 0.36 \\
\hline $\begin{array}{l}\text { Mean days of use of any acute headache medication per month at } \\
\text { baseline }\end{array}$ & $7.1 \pm 1.7$ & $7.7 \pm 1.8$ & 0.48 \\
\hline Mean HIT-6 score at baseline & $52.3 \pm 6.0$ & $53.8 \pm 6.3$ & 0.17 \\
\hline Mean MIDAS score at baseline (score; grade) & $17.8 \pm 4.7$ (III) & $18.2 \pm 3.1$ (III) & 0.55 \\
\hline
\end{tabular}

Quantitative parameters are presented as mean \pm SD. Statistical analysis was performed by paired two-tailed Student's test to compare the means at the baseline between the two groups. A fisher's exact test was performed for the male-female proportions between groups. The population included all the patients who underwent randomization and received at least one dose of a trial regimen $(n=129)$.

Table 1; Baseline Characteristics of the Patients, According to Trial Group

\begin{tabular}{|c|c|c|}
\hline & Mig-RL $(n=65)$ & Placebo $(n=64)$ \\
\hline \multicolumn{3}{|l|}{ Primary end point } \\
\hline \multicolumn{3}{|l|}{ Average no. of migraine days per month } \\
\hline Mean value, weeks 9-12 & $5.7 \pm 2.5$ & $6.7 \pm 2.0$ \\
\hline Mean change from baseline, weeks 9-12 & $-2.5 \pm 0.4$ & $-1.9 \pm 0.4$ \\
\hline Difference vs. placebo & $-0.6 \pm 0.4^{*}$ & \\
\hline \multicolumn{3}{|l|}{ Secondary end points } \\
\hline \multicolumn{3}{|l|}{ Average no. of migraine days per month } \\
\hline Mean value, weeks $1-4$ & $7.4 \pm 2.3$ & $7.7 \pm 1.7$ \\
\hline Mean change from baseline, weeks 1-4 & $-0.9 \pm 0.4^{\infty}$ & $-1.0 \pm 0.4^{2}$ \\
\hline Difference vs. placebo & $0.1 \pm 0.4$ & \\
\hline Mean value, weeks 5-8 & $6.1 \pm 2.3$ & $6.9 \pm 1.8$ \\
\hline Mean change from baseline, weeks 5-8 & $-2.3 \pm 0.3^{2000}$ & $-1.7 \pm 0.4$ \\
\hline Difference vs. placebo & $-0.6 \pm 0.4^{*}$ & \\
\hline $\begin{array}{l}\geq 50 \% \text { Reduction in average no. of headache days per month, weeks } 9-12 \\
- \text { no. of patients }(\%)\end{array}$ & $11(16.9)$ & $7(10.8)$ \\
\hline Number Needed to Treat (NNT) & 16.3 & \\
\hline $\begin{array}{l}\geq 30 \% \text { Reduction in average no. of headache days per month, weeks } 9-12 \\
- \text { no. of patients }(\%)\end{array}$ & $33(49)^{*}$ & $21(32)$ \\
\hline NNT & 5.9 & \\
\hline \multicolumn{3}{|l|}{ Days of use of any acute headache medication per month } \\
\hline Mean change from baseline, weeks 9-12 & $-2.7 \pm 0.3$ & $-1.8 \pm 0.4$.000 \\
\hline Difference vs. placebo & $-0.9 \pm 0.4^{*}$ & \\
\hline HIT-6 score & & \\
\hline
\end{tabular}




\begin{tabular}{|c|c|c|}
\hline Mean change from baseline, weeks 9-12 & $-3.9 \pm 1.2^{*}$ & $-2.5 \pm 1.1^{\text {a }}$ \\
\hline Difference vs. placebo & $-1.4 \pm 1.2^{*}$ & \\
\hline MIDAS score & & $-4.0 \pm 0.6^{\text {Mac }}$ \\
\hline Mean change from baseline, weeks 9-12 & $-5.9 \pm 0.6^{*}$ & $-1.9 \pm 0.9^{*}$ \\
\hline Difference vs. placebo & \\
\hline
\end{tabular}

Parameters are presented as mean \pm SD. Statistical analysis was performed by paired two-tailed Student's test for comparisons with baseline within

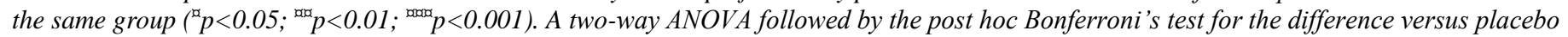
$\left({ }^{*} p<0.05 ;{ }^{* *} p<0.01 ;{ }^{* * *} p<0.001\right)$.

\section{Table 2: Primary and secondary End Points}

\section{Secondary End Points}

Weeks 1-4 of treatment did not show any significant difference between the Mig-RL group and the placebo group (Table 2). However, during the weeks 5-8, Mig-RL demonstrated statistically significant reduction from baseline in the frequency of migraine days compared to placebo $(-0.6 \pm 0.4$ [95\% confidence interval (CI) -1.20 to -0.04 ], $p=0.02$ vs placebo), similarly to weeks 9-12.

The analysis of secondary endpoints showed that $16.9 \%$ of patients in the Mig-RL group experienced a reduction of at least $50 \%$ in the number of migraine days per month during weeks $9-12$, compared to $10.8 \%$ in the placebo group (difference from placebo $[95 \% \mathrm{CI}]$ of $5.9 \%[-5.91 \%$ to $17.88 \% ; p=0.06]$ ) with the NNT (numbers need to treat) at 16.71 . The $\geq 30 \%$ migraine responder rate for weeks $9-12$ was $49 \%$ for Mig-RL and $32 \%$ for placebo (difference from placebo [95\% CI] of $18 \%[1.22 \%$ to $34.69 \% ; p=0.04]$ ) with the NNT at 5.56 .

The use of crisis treatments per day was also analysed during the weeks 9-12 and compared to placebo. It revealed a decrease of -2.7 $( \pm 0.3)$ days for the Mig-RL group and $-1.8( \pm 0.4)$ days for the placebo group, with a difference between both groups of $0.9 \pm 0.4$ ( $p=0.03$ [95\% (CI) -1.20 to $0.04])$.

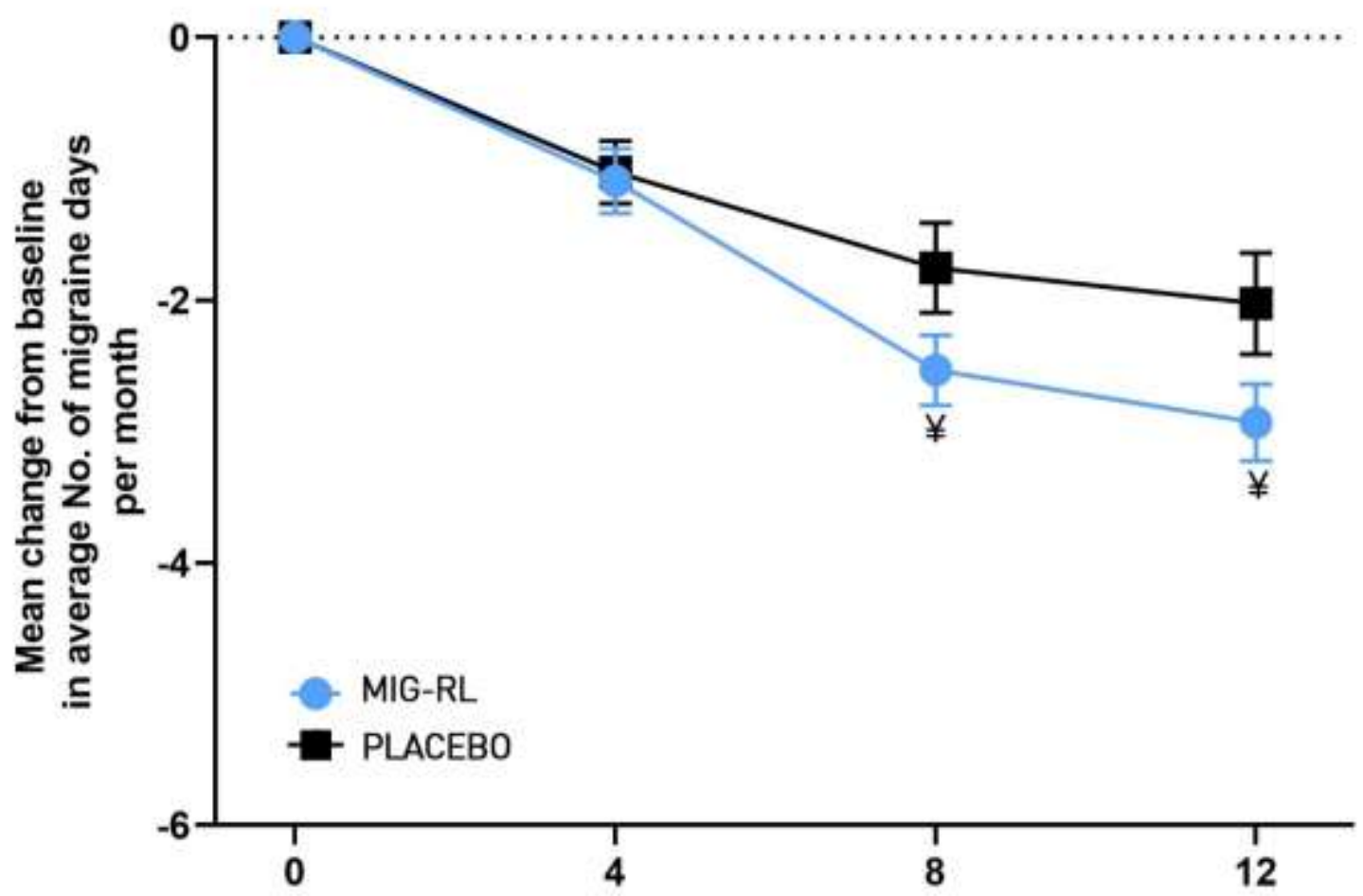

\section{Figure 2: Evolution of the migraine days between Mig-RL and Placebo}

Evolution from baseline in the average number of migraine days per month during the 12-week period after the first dose of the trial regimen in Mig-RL and placebo treated patients. $¥ p<0.05$ between Mig-RL and placebo.

HIT-6 scores indicated a decrease of $-3.9 \pm 1.2$ points in the Mig-RL group and $-2.5 \pm 1.1$ points in the placebo group compared to the baseline. The difference between both groups was $-1.4 \pm 1.2$ points $(p=0.016[95 \%$ (CI) -5.6 to -0.46$])$. The MIDAS score followed the same pattern, with a decrease in the Mig-RL group of $-5.9 \pm 0.6$ days versus $-4.0 \pm 0.6$ days in the placebo group, a difference of $1.9 \pm 0.9(p=0.045$ [95\% (CI) -2.69 to $0.03])$.

\section{Safety and adverse events}

A total of 65 patients received $\geq 1$ dose of Mig-RL and among them 59 patients received the entire treatment ( 91 doses). $10 \%$ of patients in the Mig-RL group experienced either constipation, heartburn, diarrhoea or dizziness (Table 3). Eight percent of patients in the placebo group experienced side effects such as nausea, heartburn, bloating or vomiting. No significant difference was found between the two groups.

Additional physical examination (Table 4) did not reveal any change of the vital signs or other health parameters in Mig-RL-treated or placebo- 
treated patients (throughout the trial period or after 12 weeks of treatment).

\begin{tabular}{|c|c|c|c|}
\hline & Mig-RL $; \mathbf{n},(\%)$ & Placebo $; \mathbf{n},(\%)$ & $\boldsymbol{p}$-value \\
\hline Constipation & $1(1.7 \%)$ & 0 & $\mathrm{~ns}$ \\
\hline Nausea & 0 & $1(1.7 \%)$ & $\mathrm{ns}$ \\
\hline Heartburn & $3(5.1 \%)$ & $1(1.7 \%)$ & $\mathrm{ns}$ \\
\hline Vomiting & 0 & $1(1.7 \%)$ & $\mathrm{ns}$ \\
\hline Upset stomach & 0 & $1(1.7 \%)$ & $\mathrm{ns}$ \\
\hline Diarrhoea & $1(1.7 \%)$ & 0 & $\mathrm{~ns}$ \\
\hline Dizziness & $1(1.7 \%)$ & 0 & $\mathrm{~ns}$ \\
\hline Bloating & 0 & $1(1.7 \%)$ & $\mathrm{ns}$ \\
\hline Total & $\mathbf{6}(\mathbf{1 0 \%})$ & $\mathbf{5 ( 8 \% )}$ & $\mathrm{ns}$ \\
\hline
\end{tabular}

Shown above is the data collected during the double-blind, placebo-controlled intervention period. The safety evaluation included all the patients who underwent randomization and received at least one dose of a trial regimen. Statistical analysis was performed with Chi-square test for comparison of adverse events between the two groups. "ns": not significant

Table 3: Adverse Events

\begin{tabular}{|c|c|c|c|c|}
\hline & & Mig-RL & Placebo & p-value \\
\hline \multirow{2}{*}{ SBP } & BL & $120.4 \pm 3.2$ & $121.4 \pm 3.3$ & $\mathrm{~ns}$ \\
\hline \multirow{2}{*}{ DBP } & After 12 weeks & $121.8 \pm 2.2$ & $121.6 \pm 2.1$ & $\mathrm{~ns}$ \\
\cline { 2 - 5 } & BL & $80.5 \pm 2.9$ & $81.6 \pm 3.2$ & $\mathrm{~ns}$ \\
\cline { 2 - 5 } & After 12 weeks & $81.4 \pm 2.3$ & $81.9 \pm 2.2$ & $\mathrm{~ns}$ \\
\hline \multirow{2}{*}{ PR } & BL & $76.2 \pm 5.4$ & $76.5 \pm 5.3$ & $\mathrm{~ns}$ \\
\cline { 2 - 5 } & After 12 weeks & $77.5 \pm 4.6$ & $77.1 \pm 4.8$ & $\mathrm{~ns}$ \\
\cline { 2 - 5 } & BL & $15.5 \pm 2.3$ & $15.7 \pm 2.2$ & $\mathrm{~ns}$ \\
\hline \multirow{2}{*}{$\mathbf{T}^{\circ} \mathbf{C}$} & After 12 weeks & $15.3 \pm 1.8$ & $15.2 \pm 1.9$ & $\mathrm{~ns}$ \\
\cline { 2 - 5 } & BL & $36.8 \pm 0.2$ & $36.9 \pm 0.2$ & $\mathrm{~ns}$ \\
\cline { 2 - 5 } & After 12 weeks & $36.9 \pm 0.2$ & $36.8 \pm 0.2$ & $\mathrm{~ns}$ \\
\hline
\end{tabular}

Shown above is the data collected during the double-blind, placebo-controlled intervention period. The safety evaluation included all the patients who underwent randomization and received at least one dose of a trial regimen. SBP: Systolic blood pressure (mmHg); DBP: Diastolic blood pressure ( $\mathrm{mmHg}$ ); PR: Pulse rate (beat/min); RR: Respiratory rate (breath/min), $T^{\circ}:$ Axillary body temperature (Celsius). Statistical analysis was performed by repeated two-way ANOVA for comparison between Mig-RL and placebo at baseline (BL) and after 12 weeks of treatment. "ns": not significant

Table 4 : Health parameters

\section{Discussion}

These results corroborate the observations previously made on an equivalent Mig-RL formulation, where the combination of plants reduced the frequency of migraine attacks by $61.7 \%$ over a three-month treatment period in an uncontrolled open study [20]. However, comparison between the present study and the previous one should be limited. First of all, the treatment strategy is different (once a day $v s$ twice a day in the previous trial). Second of all, there is a significant difference in the number of patients (only 14 in the first prospective study, and the absence of a control group, could be a source of bias [20]. Therefore, the present report shows more modest, but more reliable results. However, these results support the conclusions of the meta-analysis of Wider and his colleagues on the efficacy of Tanacetum parthenium in the prophylactic treatment of migraine [14]. Feverfew Mig-99 $\mathrm{CO}_{2}$-extract enriched with parthenolide showed a 2.8-day reduction in migraine days per month in the treated group of a randomized, double-blind, placebo-controlled study [25]. MigRL treatment also has a high parthenolide content $(0.75 \mathrm{mg}$ per day) and its presence may explain its beneficial role in preventing migraine attacks. In fact, studies that do not show the efficacy of Tanacetum parthenium rarely have a dosed parthenolide level. However, it is now well established that the biological properties of feverfew are mainly due to the presence of parthenolide [11]. The extraction methods but also the sourcing of the plant (origin, harvest date, storage condition) and the parts used, are important conditions for optimizing the concentration of parthenolides in the extract [12].
Propranolol is the most common preventive treatment for migraines [26]. Studies have shown that it effectively reduces the number of attacks per month by 1.3 (-2.0 to -0.62) days after 12 weeks of treatment [27]. Topiramate, another common preventive treatment, reduces the amount of migraine days per month by 0.9 (-1.3 to -0.39$)$ [27]. However, like most preventive treatments, topiramate is also not a migraine-specific treatment. Large daily doses of these non-specific treatments can cause many adverse effects [28]. Today, some patients prefer natural treatment rather than synthetic drugs and Tanacetum parthenium enriched with parthenolide may be a help for these patients. Results of this study show that associating Tanacetum parthenium and Salix Alba is slightly effective compared to similar treatment but may represent a natural and safe additional help [27].

The mechanism of action of Tanacetum parthenium in migraine prophylaxis is potentially related to the antispasmodic activity on smooth muscle vessels [29] and the anti-serotoninergic effect of parthenolide on blood platelets [13], [30]. It also has anti-inflammatory properties, resulting mainly from the inhibition of pro-inflammatory cytokines and interleukins. This inhibitory activity could be due to an action on the NF$\kappa \mathrm{B}$ pathway [31]. Another more recent theory suggests that parthenolide desensitizes the TRPA1 receptor in nociceptive neurons of the trigeminovascular system [32]. Therefore, evaluating the role of Tanacetum parthenium alone or in association with Salix alba on TRPA1 receptor desensitization, could provide a better understanding of the mode of action of Mig-RL. 
The present investigation shows that the combination of these two plants, probably with additive or synergistic pharmacological properties, can act together to prevent migraine attacks. The migraine prophylaxis properties of Salix alba were never clinically evaluated as all the studies were directed to study analgaesic properties of its active ingredient, the salicylic acid or aspirin [33]. Salicylate derivatives, particularly salicin, have anti-inflammatory properties in Salix alba and its efficacy has been demonstrated for chronic joint and muscle pain [34]. Its efficacy is believed to be caused by the inhibition of pro-inflammatory cytokines, TNF- $\alpha$, COX-2 and an action on the NF- $\kappa$ B pathway [35]. Other authors have also demonstrated the modulating effect of salicin on serotonin [36]. In the management of migraines, aspirin has only been evaluated as a crisis treatment because of its important digestive side effects [37]. It has never been proven to be effective as a prophylactic treatment [38]. The anti-inflammatory properties of Salix alba and its probable serotoninmodulating capacities may contribute synergistically to enhancing migraine prophylaxis properties of Tanacetum parthenium [39]. Whether higher migraine preventive properties of the association of plants compared to Tanacetum parthenium alone are due to additive or synergistic, analgaesic or anti-inflammatory action of Salix alba, is not clear and require further investigation.

Tanacetum parthenium combined with Salix alba were tested in an in vitro mouse model of migraine showed invasive cortical depression [17], a phenomenon potentially implicated in the appearance of migraine auras [40]. Results show a greater decrease in oxidative stress, inflammation and serotonin release when these plants are combined.

Only a few isolated and minor side effects were reported and overall MigRL was well tolerated by patients. Five percent of patients experienced heartburn-type side effects which may be due to the presence of salicin. The safety profile of Tanacetum parthenium is also corroborated by its use in the paediatric population (at a dose higher than in the present study) without any severe side effect [41]. In recent years, new specific treatments have been developed, such as monoclonal antibodies which target the CGRP or its receptor [42], representing a major advance in migraine prophylaxis but the cost of these treatments may limit its widespread use. This clinical trial shows that Mig-RL, a combination of Tanacetum parthenium and Salix alba, could provide a safe additional help to the prophylactic treatment of migraine attacks.

We acknowledge that a major limitation of this study is the large number of patients lost even before the randomization process, thus reducing the groups to a statistically acceptable minimum. The absence of other clinical studies for this association of plants in the treatment of migraine also limits the interpretation of the results. These are a first orientation which opens new perspectives in the prophylactic treatments of migraine.

\section{Conclusion}

We have shown the therapeutic effect of an association of Tanacetum parthenium with willow bark. The main active ingredients, parthenolide and salicin respectively, are the key components that may explain this effect in the prevention of migraines [39]. Unfortunately, the oral administration results in a higher degradation of the active ingredients through the digestive system. We are currently working on the development of a nasal spray form of injection to avoid a too important degradation of the active ingredients.

\section{Acknowledgements:}

The design and conduct of the trial and data analysis were supported by a grant from VITROBIO \& NATURVEDA SAS (ZAC de Lavaur 63500 ISSOIRE, France), the manufacturer of Mig-RL. We thanks the patients who participated in this trial and their families; all investigators, site personnel and coordinating investigators. We also strongly thanks Dr. Xavier MOISSET (CHU Clermont-Ferrand, France) for his precious help in writing the article and Dr. GREMEAU-RICHARD Christelle (Neurodol INSERM 1107, France) for her corrections to the protocol.

\section{References}

1. P. J. Goadsby, P. R. Holland, M. Martins-Oliveira, J. Hoffmann, C. Schankin, et S. Akerman, « Pathophysiology of Migraine: A Disorder of Sensory Processing », Physiol Rev, vol. 97 , no 2, p. 553-622, avr. 2017,

2. Headache Classification Committee of the International Headache Society (IHS) The International Classification of Headache Disorders, 3rd edition », Cephalalgia, vol. 38, no 1, p. 1-211, janv. 2018,

3. G. Demarquay, A. Ducros, A. Montavont, et F. Mauguiere, « Migraine with brainstem aura: Why not a cortical origin? », Cephalalgia, vol. 38, no 10, p. 1687-1695, sept. 2018,

4. T. Vos et al., " Global, regional, and national incidence, prevalence, and years lived with disability for 328 diseases and injuries for 195 countries, 1990-2016: a systematic analysis for the Global Burden of Disease Study 2016 », The Lancet, vol. 390, no 10100, p. 1211-1259, sept. 2017,

5. M. T. Minen et al., « Migraine and its psychiatric comorbidities », J Neurol Neurosurg Psychiatry, vol. 87, no 7, p. 741-749, juill. 2016,

6. M. Lantéri-Minet, D. Valade, G. Geraud, C. Lucas, et A. Donnet, «Revised French guidelines for the diagnosis and management of migraine in adults and children », Revue neurologique, vol. 169, déc. 2012,

7. R. B. Lipton, S. C. Marcus, A. R. Shewale, D. W. Dodick, H. N. Viswanathan, et J. A. Doshi, « Acute treatment patterns in patients with migraine newly initiating a triptan », Cephalalgia, vol. 40, no 5, p. 437-447, avr. 2020,

8. Gi. Géraud, Les céphalées en 30 leçons, ELSEVIER / MASSON. Consulté le: juin 22, 2021. [En ligne]. Disponible sur:

9. G. D'Andrea, S. Cevoli, et D. Cologno, « Herbal therapy in migraine », Neurological Sciences, vol. 35 Suppl 1, p. 135-40, mai 2014,

10. A. L. Lopresti, S. J. Smith, et P. D. Drummond, « Herbal treatments for migraine: A systematic review of randomisedcontrolled studies », Phytotherapy Research, vol. 34, no 10, p. 2493-2517, 2020,

11. F. Maggi, «Feverfew (Tanacetum parthenium(L.) Sch.Bip.) », in Nonvitamin and Nonmineral Nutritional Supplements, Elsevier., 2019, p. 223-225.

12. S. Heptinstall, D. V. Awang, B. A. Dawson, D. Kindack, D. W. Knight, et J. May, « Parthenolide content and bioactivity of feverfew (Tanacetum parthenium (L.) Schultz-Bip.). Estimation of commercial and authenticated feverfew products », J Pharm Pharmacol, vol. 44, no 5, p. 391-395, mai 1992,

13. A. Pareek, M. Suthar, G. S. Rathore, et V. Bansal, « Feverfew (Tanacetum parthenium L.): A systematic review », Pharmacogn Rev, vol. 5, no 9, p. 103-110, 2011,

14. B. Wider, M. H. Pittler, et E. Ernst, «Feverfew for preventing migraine », Cochrane Database of Systematic Reviews, no 4, 2015 ,

15. R. Freund, P. Gobrecht, D. Fischer, et H.-D. Arndt, « Advances in chemistry and bioactivity of parthenolide », Natural Product Reports, vol. 37, nov. 2019,

16. J. Nawrot et al., " The Anti-Serotonin Effect of Parthenolide Derivatives and Standardised Extract from the Leaves of Stizolophus balsamita », Molecules, vol. 24, no 22, Art. no 22, janv. 2019, 
17. V. di Giacomo et al., " Multiple pharmacological and toxicological investigations on Tanacetum parthenium and Salix alba extracts: Focus on potential application as antimigraine agents », Food and Chemical Toxicology, vol. 133, p. 110783, nov. 2019

18. D. Pandey, R. Ramamoorthy, R. Mahesh, et R. Raghuraman, « Depressant-like effects of parthenolide in a rodent behavioural antidepressant test battery », The Journal of pharmacy and pharmacology, vol. 60, p. 1643-50, janv. 2009,

19. E. Ernst et M. H. Pittler, « The efficacy and safety of feverfew (Tanacetum parthenium L.): an update of a systematic review », Public Health Nutr, vol. 3, no 4A, p. 509-514, déc. 2000,

20. R. Shrivastava, J. C. Pechadre, et G. W. John, « Tanacetum parthenium and Salix alba (Mig-RL) combination in migraine prophylaxis: a prospective, open-label study », Clin Drug Investig, vol. 26, no 5, p. 287-296, 2006,

21. W. F. Stewart, A. Shechter, et B. K. Rasmussen, « Migraine prevalence. A review of population-based studies », Neurology, vol. 44, no 6 Suppl 4, p. S17-23, juin 1994.

22. E. Loder, R. Burch, et P. Rizzoli, « The 2012 AHS/AAN guidelines for prevention of episodic migraine: a summary and comparison with other recent clinical practice guidelines », Headache, vol. 52, no 6, Art. no 6, juin 2012,

23. A. F. H. Smelt, W. J. J. Assendelft, C. B. Terwee, M. D. Ferrari, et J. W. Blom, «What is a clinically relevant change on the HIT-6 questionnaire? An estimation in a primary-care population of migraine patients », Cephalalgia, vol. 34, no 1, p. 29-36, janv. 2014,

24. R. Lipton, P. Desai, S. Sapra, D. Buse, K. Fanning, et M. Reed, « How much change in Headache-Related Disability is clinically meaningful? Estimating minimally important difference or change in MIDAS using data from rhe AMPP study », présenté à Boston American Headache Society Annual Meeting, Boston, 2017.

25. H. Diener, V. Pfaffenrath, J. Schnitker, M. Friede, et H.-H. H. Zepelin, «Efficacy and Safety of $6.25 \mathrm{mg}$ t.i.d. Feverfew CO2Extract (MIG-99) in Migraine Prevention - A Randomized, Double-Blind, Multicentre, Placebo-Controlled Study », Cephalalgia, vol. 25, no 11, p. 1031-1041, nov. 2005,

26. S. D. Silberstein, «Preventive treatment of headaches », Curr Opin Neurol, vol. 18, no 3, p. 289-292, juin 2005,

27. J. L. Jackson et al., «A Comparative Effectiveness MetaAnalysis of Drugs for the Prophylaxis of Migraine Headache », PLoS One, vol. 10, no 7, p. e0130733, 2015,

28. S. D. Silberstein, «Preventive Migraine Treatment », Continuum (Minneap Minn), vol. 21, no 4 Headache, p. 973989, août 2015,

29. A. Orona-Ortiz et al., « Inhibition of phenylephrine- or serotonin-induced rat aorta contractions by mexican feverfew (<em>Tanacetum parthenium</em> (L.) Schultz-Bip). », Revista Latinoamericana de Química, vol. 46, no 2-3, Art. no 2 $-3,2018$.
30. S. Mittra, A. Datta, S. Singh, et A. Singh, « 5Hydroxytryptamine-inhibiting property of Feverfew: Role of parthenolide content », Acta pharmacologica Sinica, vol. 21, p. 1106-14, janv. 2001.

31. A. J. García-Piñeres et al., " Cysteine 38 in p65/NF-kappaB plays a crucial role in DNA binding inhibition by sesquiterpene lactones », J Biol Chem, vol. 276, no 43, p. 39713-39720, oct. 2001,

32. S. Materazzi et al., «Parthenolide inhibits nociception and neurogenic vasodilatation in the trigeminovascular system by targeting TRPA1 channel », Pain, vol. 154, no 12, p. 10.1016/j.pain.2013.08.002, déc. 2013,

33. C. P. Baena, R. C. D'Amico, H. Slongo, A. R. Brunoni, A. C. Goulart, et I. Benseñor, "The effectiveness of aspirin for migraine prophylaxis: a systematic review », Sao Paulo Med. J., vol. 135, p. 42-49, févr. 2017,

34. B. Kammerer, R. Kahlich, C. Biegert, C. H. Gleiter, et L. Heide, «HPLC-MS/MS analysis of willow bark extracts contained in pharmaceutical preparations », Phytochem Anal, vol. 16, no 6, p. 470-478, déc. 2005 ,

35. G. A. Bonaterra, E. U. Heinrich, O. Kelber, D. Weiser, J. Metz, et R. Kinscherf, "Anti-inflammatory effects of the willow bark extract STW 33-I (Proaktiv(®)) in LPS-activated human monocytes and differentiated macrophages », Phytomedicine, vol. 17, no 14, p. 1106-1113, déc. 2010,

36. G. Ulrich-Merzenich et al., « Novel neurological and immunological targets for salicylate-based phytopharmaceuticals and for the anti-depressant imipramine », Phytomedicine, vol. 19, no 10, p. 930-939, juill. 2012,

37. M. Shara et S. J. Stohs, « Efficacy and Safety of White Willow Bark Extracts », Phytother. Res., vol. 29, no 8, p. 1112-1116, août 2015,

38. M. Lantéri-Minet, D. Valade, G. Geraud, C. Lucas, et A. Donnet, "Revised French guidelines for the diagnosis and management of migraine in adults and children ", Revue neurologique, vol. 169, déc. 2012,

39. R. Shrivastava, «Association de l'écorce de Saule et de la Grande Camomille: une alternative thérapeutique aux traitements de fond de la migraine ", These de doctorat, Université Clermont Auvergne (2017-2020), 2020.

40. A. Charles et K. Brennan, «Cortical Spreading DepressionNew Insights and Persistent Questions », Cephalalgia, vol. 29, no 10 , p. $1115-1124$, oct. 2009 ,

41. F. Moscano et al., «An observational study of fixed-dose Tanacetum parthenium nutraceutical preparation for prophylaxis of pediatric headache », Italian Journal of Pediatrics, vol. 45, no 1, p. 36, mars 2019,

42. F. Urs, « Neurologie: Anticorps monoclonaux anti-CGRP: des «game changers» dans la prophylaxie de la migraine? », 2019 :1, no 1, janv. 2019, Consulté le: juin 22, 2021. [En ligne]. 
(c) (†) This work is licensed under Creative Commons Attribution 4.0 License

To Submit Your Article Click Here: Submit Manuscript

DOI: $10.31579 / 2578-8868 / 192$
Ready to submit your research? Choose Auctores and benefit from:

* fast, convenient online submission

* rigorous peer review by experienced research in your field

* rapid publication on acceptance

* authors retain copyrights

* unique DOI for all articles

* immediate, unrestricted online access

At Auctores, research is always in progress.

Learn more www.auctoresonline.org/journals/neuroscience-andneurological-surgery 